Angela Million

\title{
Baukulturelle Bildung
}

Schule als Drehscheibe baukultureller Vermittlungspraxis

\section{Zusammenfassung}

Baukulturelle Bildungsangebote thematisieren den gebauten Raum und den damit verbundenen Gestaltungsprozess. Sie bieten ein soziales Bildungssetting und stärken die Selbstwirksamkeit, Kreativität und Teilhabe von Kindern und Jugendlichen. Baukulturelle Bildung ist ein multiprofessionelles Feld, in dem Baukultur als Querschnittsthema in Kooperation mit Vereinen, Stiftungen oder Museen bereits in Schule umgesetzt wird. Darüber hinaus bestehen Notwendigkeiten und Potenziale, dieses Angebot weiter auszubauen.

Schlüsselwörter: Baukulturelle Bildung, Architekturbildung für Kinder und Jugendliche, multiprofessionelles Unterrichten in Schule, phänomenologisches Lernen, Portfolio-Lernen

\section{Built Environment Education}

School as a Hub for Educational Activities on Architecture

\begin{abstract}
Built environment education programs focus on built space and the associated processes of design, planning, and co-construction. Activities offer a social educational setting and strengthen the self-efficacy, creativity, and participation of children and adolescents. Built environment education is a multi-professional field in which "Baukultur" as a cross-cutting theme is already implemented in schools in cooperation with associations, foundations, or museums. In addition, there are necessities and potentials to further expand this offer.

Keywords: built environment education, architecture education for children and adolescents, multi-professional teaching in school, phenomenological learning, portfolio-learning
\end{abstract}




\section{Baukulturelle Themen und Akteure}

Baukulturelle Bildungsinhalte lassen sich in vielen schulischen Fächern verorten. In baukulturellen Bildungsangeboten werden die Planung der Umwelt und dazugehörige Gestaltungs- und Aushandlungsprozesse thematisiert. Die gebaute Umwelt wird in ihren technischen, ästhetischen und politischen Ausprägungen und beeinflussenden Aspekten auf verschiedenen Maßstabsebenen des Gebäudes, der Nachbarschaft, der Gesamtstadt oder Region erkundet. Gegenstand der Diskussion sind das baukulturelle Erbe, die Geschichte und das nachhaltige Weiterbauen von Gebäuden und Stadtstrukturen. Damit bietet Baukultur auch zahlreiche Anknüpfungspunkte für eine fächerübergreifende Bildungsarbeit. Im Folgenden wird die baukulturelle Bildungsarbeit in der thematischen Breite, vor allem aber in der Verknüpfung mit schulischer Bildung vorgestellt. Zurückgegriffen wird auf eigene Forschung (Million et al., 2019), in welcher die baukulturelle Bildungspraxis - darunter auch die schulischen Angebote - und die Sichtweise der teilnehmenden Kinder und Jugendlichen untersucht worden sind.

Dabei muss vorweg betont werden, dass die baukulturelle Bildungspraxis im deutschsprachigen Raum, aber auch international, traditionell vor allem durch Architekt ${ }^{\star}$ innen und Stadt- sowie Landschaftsplaner*innen entwickelt worden ist (vgl. UIA Built Environment Education Network, 2016) - jenseits der schulischen Bildung. In der Praxis hingegen hat sich vielerorts ein multiprofessionelles Feld an Anbieter*innen aus den Bereichen Kunst, Handwerk und Pädagogik (von Schulpädagogik über $\mathrm{Mu}$ seumspädagogik bis Sozialpädagogik) herausgebildet. Die multiprofessionellen Anleiter*innen sind ihrerseits wiederum in verschiedenen Anbieterstrukturen verortet und organisiert, die ebenfalls die Grundlage für vielfältige Kooperationen im Bereich der baukulturellen Bildung bieten und in denen Schulen durchaus wichtige Partnerinnen darstellen (Million \& Hock, 2020).

\section{Baukulturelle Bildung im Schulunterricht}

Auch wenn die Bezüge zu baukulturellen Themen vielfältig sind, werden diese im Schulunterricht häufig eher punktuell aufgegriffen: Vielfach geht es um theoretische Kenntnisse zu Stadtentwicklungsfragen im Fach Geografie oder auch um Stil-Epochen der Architektur, die im Kunstunterricht vermittelt werden, ergänzt um praktische Fähigkeiten, wie technisches Zeichnen oder Modellbau (Million et al., 2019, S. 12f.; vgl. hierzu Abb. 1 im Anhang). Baukultur eignet sich vor allem als Querschnittsthema, in dem neben gestalterischen Aspekten mathematische, aber auch politische und wirtschaftliche Zusammenhänge behandelt werden können. Explizit wird international wie national die Verknüpfung mit den MINT-Fächern gesucht und eingefordert. Bei der Behandlung von scheinbar wenig komplexen Themen, wie 
z. B. beim Thema „Hochhäuser“, können und müssen genauso wie bei der Behandlung brisanter gesellschaftspolitischer Fragen, wie z. B. bei der Entwicklung des Wohnens, des öffentlichen Raums oder des Klimawandels, regelmäßig die oben genannten Fächer eingebunden werden (vgl. Kursmaterial für Klimawandel, Stadtumbau und Zusammenleben, Landesinstitut für Lehrerbildung und Schulentwicklung et al., 2013; Wüstenrot Stiftung, 2017). Außerdem kann auf thematisch einschlägige Schulbücher von Verlagen, z. B. von Klett oder Schöningh, zurückgegriffen werden. Bislang gibt es jedoch keinen Überblick darüber, wie regelmäßig und wie thematisch begrenzt diese Inhalte im Unterricht behandelt werden. Doch je nach Bundesland und Schulform werden baukulturelle Themen auf freiwilliger Basis vor allem in Projektwochen als Querschnittsthemen aufgegriffen. Am Ammersee-Gymnasium in Dießen (Bayern) kann sogar ein ganzes „Architektursemester“ belegt werden (Million et al., 2019, S. 38-43).

Baukulturelle Bildung im schulischen Unterricht ist auch international ein Thema. In einer Studie mit Lehrer*innen, Schulleiter*innen und (Schul-)Verwaltungsmitarbeiter*innen in London wurde deutlich, dass eine hohe Nachfrage nach (bau-) kultureller Bildung (architecture education) besteht. Gleichzeitig wurde auch offensichtlich, dass es an Informationen über mögliche Partner*innen in der Bildungsarbeit und über Methoden fehlte. Ebenso wurde die Notwendigkeit gesehen, ortsansässige Praktiker*innen in den Unterricht einzubeziehen (vgl. Kendall et al., 2007a, 2007b). In einer anderen Studie zur Frage der Verankerung von baukultureller Bildung an Schweizer Schulen wird zwar festgestellt, dass Baukultur sowohl in den Curricula der Pädagogischen Hochschulen als auch in den Lehrplänen der Schulen in allen geeigneten Fächern vorgesehen ist. Doch eine Befragung von Pädagog^innen in den Schulen zeigte, dass das Thema eher vereinzelt und ohne fächerübergreifende $\mathrm{Zu}$ sammenhänge bearbeitet wird (Gaus-Hegner et al., 2019, S. 45). Es ist anzunehmen, dass ähnliche Befunde für den schulischen Bereich in Deutschland zutreffen. Hier finden sich in den jeweiligen Lehrplänen nur vereinzelte Hinweise oder „überhaupt keine Hinweise zur Einbeziehung des Themenbereichs: Architektur Baukultur“ (Wüstenrot Stiftung, 2010, S. 9). Allerdings zeigt die aktuelle Praxis, dass Schulen, die mit anderen Akteur*innen als Partner ${ }^{\star}$ innen zusammenarbeiten, baukulturelle Themenstellungen in den Unterricht erfolgreich integrieren. Ob dies jedoch die Regel oder die Ausnahme im Schulbetrieb ist, ist ungeklärt.

\section{Schule als Drehscheibe baukultureller Vermittlungspraxis}

Gerade in den letzten Jahren haben sich im Zuge der Ganztagsschulkonzepte zusätzliche Möglichkeiten ergeben, baukulturelle Angebote sowohl innerhalb als auch außerhalb der Unterrichtszeit durchzuführen, zum Beispiel in Kooperation mit Vereinen, Stiftungen, Hochschulen, Museen oder Berufsverbänden. Dies knüpft an den 
ersten Initiativen aus den 1970er-Jahren und dem Engagement der Berufsverbände der weltweit verbreiteten Architekt*innenkammern und deren Programme architects in schools an. Heute werden Architekt*innen und Planer*innen an Schulen als Expert ${ }^{\star}$ innen für die Fragen des Bauens und Planens vermittelt und gemeinsam mit Pädagog*innen gestalten sie Nachmittags-AGs im Rahmen der Ganztagsschulbetreuung wie auch Projekttage oder -wochen. Die Architekt*innenkammern (AK) loben ebenso Schulwettbewerbe aus (z. B. „Architektur für neues Lernen“ der Landesarbeitsgemeinschaft Bayern e.V. und der AK Bayern oder „Mein Bauhaus - Meine Moderne“ der AK Berlin/Brandenburg). Außerdem entwickeln auch sie Unterrichtsmaterialien (z.B. „Architektur - Bausteine für den Themenplan - Eine Information für Lehrerinnen und Lehrer an den allgemeinbildenden Schulen in Brandenburg“").

Darüber hinaus bieten Museen relevante, auf Baukultur bezogene Programme für Schulklassen an, die gerne und intensiv genutzt werden. Hinzu kommen - wie schon angesprochen - Vereine und Einzelaktive, die als Expert*innen mit Schulen zusammenarbeiten. Ein Blick auf die Mitglieder des "Netzwerks Baukulturelle Bildung“ der Bundesstiftung Baukultur (2021) zeigt, wie breit das Spektrum der Beteiligten wie auch der Angebotsformate ist; letzteres reicht von Bauworkshops, Exkursionen und Stadtspaziergängen bis hin zu konkreten urbanen Interventionen oder der Mitgestaltung von Schulhöfen. Beispielsweise ist der Verein „Jugend Architektur Stadt e. V.“ (JAS, 2021) derzeit häufig mit seinen Formaten beteiligt, wenn Schüler*innen im Rahmen von Neubau und Umbau von Schulen mitarbeiten. Letzteres ist zudem ein Beispiel für den fließenden Übergang zwischen baukulturellen Bildungsaktivitäten und der Beteiligung von Kindern und Jugendlichen an baulichen Veränderungen; in beiden Fällen wird mit gleichen Methoden gearbeitet (Edelhoff et al., 2019).

In dem eingangs angesprochenen transdisziplinären Forschungsprojekt von Million et al. (2019) wurde untersucht, wie Heranwachsende baukulturelle Angebote erleben und in ihre alltägliche Lebenswelt einbeziehen. Befragt wurden Kinder und Jugendliche zwischen acht und 18 Jahren aus unterschiedlichen Herkunftsmilieus. Unter den untersuchten Angeboten zu baukultureller Bildung waren auch zwei Angebote mit Partner*innen in Schulen und das schon erwähnte Architektursemester am Ammersee-Gymnasium. In den Gesprächen mit den Kindern und Jugendlichen beurteilten diese - vergleichend und quer zu allen untersuchten Angeboten - die Angebote zu baukultureller Bildung und deren Akteur ${ }^{\star}$ innen positiv. Sie schätzten vor allem das „selber machen“ und „selbst gestalten“ in einem sozialen Setting unter Anleitung von Fachleuten, die sie als überaus unterstützend und fachlich kompetent wahrgenommen hatten. So haben sie das Setting beispielsweise als „entspannt“, „locker“ und „fröhlich" beschrieben. Sie mochten die weniger standardisierten Abläufe der Angebote, das flexible Handling von Gruppen- und Einzelarbeit und die erfahrene gegenseitige Unterstützung, Hilfsbereitschaft und den Austausch mit den Peers in durchaus auch herausfordernden Gruppenprozessen (Million et al., 2019, S. 79 ff.). Häufig wird baukulturelle Bildung mit dem regulären Unterricht verglichen. Dabei wird deutlich, dass 
das Setting Schule oder Unterricht sich positiv, aber auch negativ auf die individuelle baukulturelle Lernerfahrung auswirken kann und dass sich daraus Implikationen für die baukulturelle kooperative Bildungspraxis ergeben:

\begin{abstract}
"Schule ist besonders gut zur Vermittlung theoretischer Kenntnisse geeignet und kann diese auch mit Praxis verbinden (Prinzip der Zweipunktperspektive lernen und selbst umsetzen, Gebäudestile lernen und wiedererkennen usw.); aber Vorgaben und Regeln schulischen Unterrichts wie auch Benotung sind zu berücksichtigende Faktoren und stellen eine Herausforderung sowohl für die Offenheit als auch Kreativität in der baukulturellen Bildung dar. Schule kann als Ort der baukulturellen Bildung dann nachteilig wirken, wenn das Angebot einseitig in stark formalisierte Strukturen überführt und als aufgezwungenes Thema wahrgenommen wird. Zugleich werden gerade baukulturelle Projekte in Schulen von Kindern und Jugendlichen als mit mehr Freiheit ausgestattet empfunden. Daher legen die Befragungen nahe, gerade in diesen fächerübergreifenden Unterrichtsformaten, im Wahlbereich des Fächerkanons oder in Kooperationsprojekten baukulturelle Bildungsinhalte zu stärken“ (Million et al., 2019, S. 210-211).
\end{abstract}

Zugleich hat die Studie gezeigt, wie Bildungsbenachteiligung in der baukulturellen Bildung wirksam wird. Denn Momente baukultureller Bildung finden auch in den familiären Bildungswelten statt: beim Hausbau, bei der eigenen Zimmergestaltung oder im Rahmen von Urlaubsreisen wie in der Nutzung von (digitalen) Medien. Heranwachsende in benachteiligten Settings erleben solche Momente baukultureller Bildung deutlich seltener. Daher ist die Schule als Ort, in dem ein breit gefächerter Zugang zu solchen Erfahrungen für alle Kinder und Jugendlichen ermöglicht wird, unverzichtbar.

\title{
4 Ansätze zu einer Implementierung von baukultureller Bildung in die schulischen Curricula
}

Ausgangspunkt für eine Implementierung von baukultureller Bildung in das Bildungssystem sind die schulischen Curricula. Daher wäre zunächst eine Grundlagenermittlung der Lehrpläne hilfreich, um hier schon Vorhandenes wie auch Lücken zu identifizieren. Aktuell wird hierzu eine Studie des Bundesinstituts für Bau-, Stadt- und Raumforschung durchgeführt (BBSR, 2021). Die derzeitige Kompetenzorientierung in der schulischen Bildung bietet die Möglichkeit, die handlungsorientierte Dimension der baukulturellen Bildung auch hier stärker zu nutzen. In der Praxis zeigen sich bereits Anknüpfungspunkte für aktivierendes Lernen im Rahmen baukultureller Bildung. Genauso sollten die aktuell diskutierten zukunftsorientierten Lehr- und Lernkonzepte - das phänomenologische Lernen oder das PortfolioLernen - in den Blick genommen werden. Wünschenswert wäre auch eine Weiterentwicklung der baukulturellen Bildung in den Ausbildungsgängen für pädagogische 
Berufe an Universitäten und Akademien, in denen die baukulturelle Bildung - insbesondere als Querschnittsthema für die schulische Bildung - bisher noch nicht Teil des Curriculums ist. Momentan gibt es weder eine Fachwissenschaft noch eine Fachdidaktik. Dennoch wird seit vielen Jahren ein fachdidaktisch unterlegtes Modell der Architekturvermittlung an der Bauhaus-Universität Weimar (2021) praktiziert; es könnte durchaus als Ausgangspunkt und Orientierung dienen. Auch wenn die Ausbildung der Pädagog*innen im Bereich der baukulturellen Bildung noch am Beginn steht: Die Weiterbildung von Lehrkräften in Fragen der Architektur und Baukultur hat sich in vielen Bundesländern mittlerweile etabliert und Vereine, Initiativen und die Architekt*innenkammern engagieren sich hier. Seit 2019/2020 widmen sich zudem die „Akademie der Kulturellen Bildung Remscheid“ und die „Bundesakademie für Kulturelle Bildung Wolfenbüttel e.V.“ dem Thema Baukultur.

Festzuhalten ist, dass Baukultur bzw. baukulturelle Bildung ein Querschnittsthema ist, das „zahlreiche [...] Verweise und Schnittstellen zu vielen Bildungsbereichen umfasst" (Million et al., 2019, S. 216). Diese Feststellung kann als Problem gesehen werden, weil thematische Zuständigkeiten und Abgrenzungen schwer greifbar sind, zumal viele Fächerkulturen und Fachsprachen „verschnitten“ werden müssen und dies die Komplexität des Unterrichtens und Lernens erhöht. Dennoch bietet gerade die Baukultur vielfältige Möglichkeiten für integriertes Lernen und eine ganzheitliche, multiprofessionale Bildungsarbeit. Die erwähnten Kooperationen und Partnerschaften zwischen Schule und anderen Akteurinnen zeigen, wie dies in der schulischen Praxis bereits erfolgreich ausgestaltet wird. Darüber hinaus kann bereits jetzt - auch ohne Verankerung im Curriculum - „baukulturelle Bildung über die Kulturelle Bildung und auch die Bildung für nachhaltige Entwicklung mehr als bisher in Schulen" (Million et al., 2019, S. 216) thematisiert werden. Dabei geht es am Ende vor allem um die Vermittlung der Inhalte und Kompetenzen für die Gestaltung der Umwelt, und nicht darum, Baukultur als Begriff zu verankern. Das bedeutet: Baukultur kann viel und ist Vieles, auch wenn das, was gearbeitet und gelernt wird, nicht unter diesem Begriff firmiert. Oder in den Worten eines befragten Jugendlichen:

„Ich finde auf jeden Fall den Prozess, mit der Hand zu arbeiten und so etwas zu entwerfen, sehr schön. Und gestern saß ich, auch glaube ich, an der Treppe zwei oder drei Stunden und habe da halt mit dem Cutter [gearbeitet]. Also irgendwo ist es natürlich anstrengend und viel. Aber irgendwo macht es auch wahnsinnig Spaß, das zu machen“ (Million et al., 2019, S. 76).

\section{Literatur und Internetquellen}

Architekturvermittlung an der Bauhaus-Universität Weimar. (2021). Architektur und Schule. https://www.uni-weimar.de/de/kunst-und-gestaltung/studium/lehramt-an-gymnasien/ architektur-und-schule/ 
BBSR (Bundesinstitut für Bau-, Stadt- und Raumforschung). (2021). Baukulturelle Bildung: Bestand, Bedarf, Wirksamkeit. https://www.bbsr.bund.de/BBSR/DE/forschung/pro gramme/exwost/Studien/2020/baukulturelle-bildung/01-start.html

Bundesstiftung Baukultur. (2021). Baukulturelle Bildung. https://www.bundesstiftung-bau kultur.de/baukultur-bundesweit/baukulturelle-bildung

DAM (Deutsches Architekturmuseum Stuttgart). (2021). Architekturvermittlung im DAM: Plan. Werk. Stadt. Führungen, Workshops und Projekte für Kinder und Jugendliche. https://dam-online.de/bildung/

Edelhoff, S., Fleckenstein, R., Grotkamp, B., von Jagow, B., Kataikko-Grigoleit, P., \& Million, A. (2019). Handbuch der baukulturellen Bildung. Universitätsverlag der TU Berlin. http://dx.doi.org/10.14279/depositonce-8252

Gaus-Hegner, E., Gassmann, U., Kels, S., Mosimann, P., \& Schreier, M. (2019). Baukulturelle Bildung an Schweizer Schulen. Analyse von Bestand und Bedarf. https://www.archijeu nes.ch/wp-content/uploads/2019/03/BKB-an-Schweizer-Schulen_2019.pdf

Kendall, S., Murfield, J., White, R., \& Wilkin, A. (2007a). Research into the supply and demand of built environment education in schools. DCMS. https://files.eric.ed.gov/fulltext/ ED501876.pdf

Kendall, S., Murfield, J., \& Wilkin, A. (2007b). Engaging Places: teacher research. DCMS. https://www.nfer.ac.uk/media/1621/bee03.pdf

Landesinstitut für Lehrerbildung und Schulentwicklung, Internationale Bauausstellung IBA Hamburg GmbH \& JAS WERK - Jugend Architektur Stadt gUG. (2013). Stadt neu bauen - Materialien für die Kursarbeit. https:/li.hamburg.de/publikationen/4141802/ stadt-neu-bauen/

Million, A., Coelen, T., Bentlin, F., Klepp, S., \& Zinke, C. (2019). Bildungsorte und Lernwelten der Baukultur. Momente und Prozesse baukultureller Bildung von Kindern und Jugendlichen. Wüstenrot Stiftung. ${ }^{1}$ https://wuestenrot-stiftung.de/publikationen/ bildungsorte-und-lernwelten-der-baukultur/

Million, A., \& Hock, L. (2020). Quo vadis baukulturelle Bildung? Eine Einordnung der baukulturellen Bildungspraxis. Kulturelle Bildung online. https://www.kubi-online.de/arti kel/quo-vadis-baukulturelle-bildung-einordnung-baukulturellen-bildungspraxis

Stadtbau Akademie des Museums für Stuttgart. (2021). StadtbauAkademie. https://www. stadtpalais-stuttgart.de/stadtbauakademie/stadtbauakademie

UIA Built Environment Education Network. (2016). Architecture \& Children - UIA Built Environment Education Network. https://www.architectureandchildren-uia.com/

Verein JAS Jugend Architektur Stadt e.V. (2021). JAS. http://www.jugend-architektur-stadt. de/.

Wüstenrot Stiftung. (2010). Baukultur - Gebaute Umwelt: curriculare Bausteine für den Unterricht. Wüstenrot-Stiftung. https://wuestenrot-stiftung.de/publikationen/baukul tur-gebaute-umwelt-curriculare-bausteine-fuer-den-unterricht/

Wüstenrot Stiftung. (2017). Baukultur - Gebaute Umwelt: Fächerverbindende Arbeitsblätter: der öffentliche Raum. Wüstenrot Stiftung. https://wuestenrot-stiftung.de/publikatio nen/baukultur-gebaute-umwelt-curriculare-bausteine-fuer-den-unterricht/

1 Die hier angeführten Publikationen der Wüstenrot Stiftung können über die angegebenen Links kostenlos bei der Wüstenrot Stiftung bestellt werden. 
Angela Million, Prof. Dr.-Ing., Stadtplanerin und Städtebauerin, geb. 1974, Professorin und Fachgebietsleiterin Städtebau und Siedlungswesen an der Technischen Universität Berlin, Fakultät VI Planen - Bauen - Umwelt, Institut für Stadt- und Regionalplanung (ISR).

E-Mail: million@tu-berlin.de

Korrespondenzadresse: Technische Universität Berlin, Fakultät VI Planen - Bauen - Umwelt, Institut für Stadt- und Regionalplanung (ISR), Fachgebiet Städtebau und Siedlungswesen, Hardenbergstr. 40A, 10623 Berlin 


\section{Anhang}

Abb. 1: Baukulturelle Bildung - Kindergarten und Schule als Bildungsort

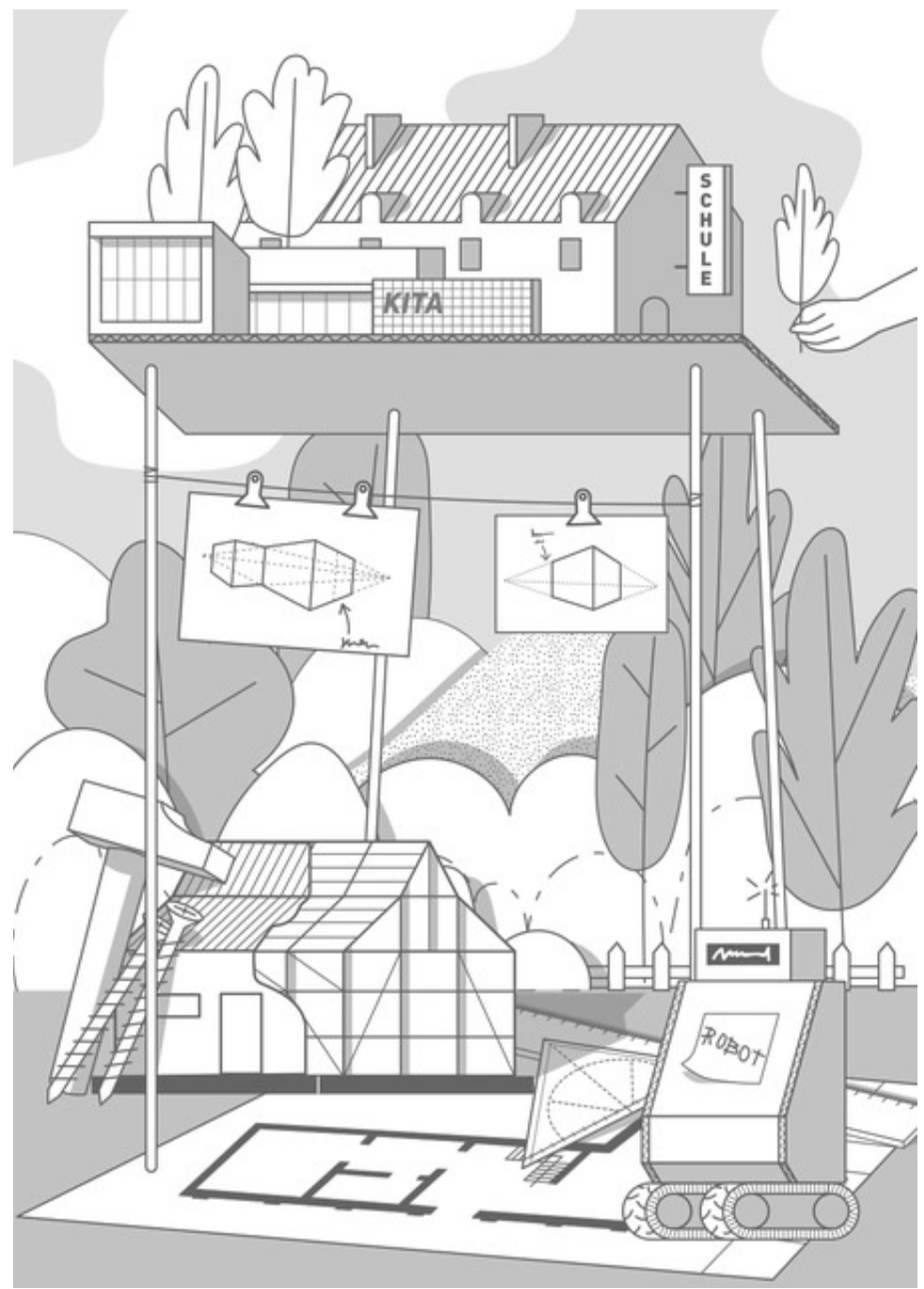

Quelle: Luca Múle

Anm.: „Interessanterweise wird baukulturelles Lernen im Kindergarten und im Schulalltag [mit Ausnahme derjenigen Befragten, die im schulischen Rahmen Baukulturangebote wahrgenommen haben] von den Befragten eher ausschnitthaft thematisiert. Im Kontext institutionalisierter Bildungssettings berichten die Befragten von punktuell erlebten baukulturellen Spiel- und Lernaufgaben, wobei diese sich in Kindergarten und Grundschule zunächst im Bereich spielerischer Bau- und Kreativtätigkeiten bewegen wie Malen, Basteln oder Bauen mit unterschiedlichen Materialien. In der weiterführenden Schule beziehen sich die Aussagen der Jugendlichen dann stärker auf strukturierte sowie formalere baukulturelle Lernaufgaben und -prozesse wie etwa räumliches Zeichnen oder Modellbau" (Million et al. 2019, S. 190 f.). 